\title{
Oncogenic function and prognostic significance of Abelson interactor 1 in hepatocellular carcinoma
}

\author{
JI-LONG WANG ${ }^{1}$, TING-TING YAN ${ }^{2}$, CHEN LONG $^{1}$ and WEN-WU CAI ${ }^{1}$ \\ ${ }^{1}$ Department of General Surgery, The Second Xiangya Hospital, Central South University, Changsha, Hunan 410011; \\ ${ }^{2}$ Xiangya School of Public Health, Central South University, Changsha, Hunan 410008, P.R. China
}

Received December 14, 2016; Accepted March 3, 2017

DOI: $10.3892 /$ ijo.2017.3920

\begin{abstract}
Aberrant expression of Abelson interactor 1 (ABI1) has been reported in multiple cancers. However, its clinical significance and potential biological roles in hepatocellular carcinoma (HCC) have not been fully elucidated. In this study, we found that ABI1 was obviously upregulated in HCC tissues compared with non-tumor tissues. Moreover, high ABI1 expression was significantly correlated with tumor size $(\mathrm{P}=0.041)$, tumor number $(\mathrm{P}<0.001)$, tumor encapsulation $(\mathrm{P}<0.001)$ and $\mathrm{BCLC}$ stage $(\mathrm{P}=0.010)$. Importantly, Kaplan-Meier survival analysis showed that increased ABI1 expression predicted shorter overall survival time $(\mathrm{P}<0.001)$ and a higher tendency of tumor recurrence $(\mathrm{P}=0.001)$ in HCC patients. Multivariate Cox regression analysis further confirmed high ABI1 expression was an independent predictor for both overall survival $(\mathrm{HR}=1.795, \mathrm{P}=0.025)$ and early recurrence $(\mathrm{HR}=1.893, \mathrm{P}=0.012)$ after surgical resection. Furthermore, in vitro studies indicated that overexpression of ABI1 induced an increase in cell proliferation, migration and invasion of HCC cells, whereas knockdown of ABI1 did the opposite. Xenograft mouse models verified the promoting effects of ABI1 on HCC growth and lung metastasis in vivo. Collectively, our findings indicated that ABI1 contributes to the development and progression of $\mathrm{HCC}$ as an oncogene and may serve as a valuable prognostic marker for $\mathrm{HCC}$ patients.
\end{abstract}

Correspondence to: Dr Ji-Long Wang, Department of General Surgery, The Second Xiangya Hospital of Central South University, 139 Renmin Middle Road, Changsha, Hunan 410011, P.R. China E-mail: wangjilong@csu.edu.cn

Abbreviations: ABI1, Abelson interactor 1; HCC, hepatocellular carcinoma; qRT-PCR, quantitative real-time polymerase chain reaction; GAPDH, glyceraldehyde 3-phosphate dehydrogenase; IHC, immunohistochemistry; mRNA, microRNA; shRNA, short hairpin RNA; PBS, phosphate buffered saline; FBS, fetal bovine serum

Key words: Abelson interactor 1, hepatocellular carcinoma, prognosis, proliferation, metastasis

\section{Introduction}

Hepatocellular carcinoma (HCC) is one of the most prevalent malignant tumors, especially in China, and has become the second leading cause of cancer-related death $(1,2)$. The lack of effective early detection and high frequency of postoperative metastasis and recurrence resulted in a very low 5 -year survival rate (3). Thus, it is essential to clarify the underlying mechanism of HCC progression, which may contribute to identification of novel targets for effective intervention.

Abelson interactor 1 (ABI1), also known as E3B1, was originally identified as Abl kinase associating protein 1 (4) and later confirmed to be one of the Bcr-Abl interactors (5). ABI1 regulated cell proliferation via the Ras small G-protein and affect actin remodeling, cell adhesion, and cell migration via $\mathrm{Rac}$ activation $(6,7)$. It also plays a central role in holding multiprotein complexes together (ABI-WAVE complex and Sos1-Eps8-ABI1 complex) $(8,9)$, which mediate a number of pathways related to cell spreading and migration (10-12). For the crucial role of ABI1 in actin reorganization and cell migration, homozygous loss of this protein leads to embryonic lethality in mice $(9,13)$. In cancers, previous studies showed that ABI1 participated in both tumor progression and tumor suppression. Initially, ABI1 has been proposed as a potential tumor suppressor based on the evidence that ABI1 gene is deleted in prostate cancer and decreased in gastric cancer and some types of leukemia (14-17). ABI1 has been reported to be overexpressed and correlated with poor prognosis in a wide variety of tumors, such as breast cancer, BCR-Abl-induced leukemia, colon cancer, HCC and ovarian cancer (18-25). These studies suggested that ABI1 may play a diverse role during cancer progression under various circumstances. Although Liu et al (25) determined the expression of ABI1 in $40 \mathrm{HCC}$ specimens, the role of ABI1 in regulating HCC progression as well as its clinical significance remain largely unexplored.

In this study, we investigated the expression of ABI1 both in human HCC tissues and cell lines. The prognostic value of ABI1 in HCC patients after curative resection was also valuated. Furthermore, we established ABI1 overexpression and knockdown stable clones in HCC cell lines and for the first time explored the functions of ABI1 in HCC cells both in vitro and in vivo assays. 


\section{Materials and methods}

Tissue samples. Paraffin-embedded HCC samples used in our study were obtained from $124 \mathrm{HCC}$ patients who underwent hepatic resection at the Second Xiangya Hospital of Central South University between March 2009 and October 2011. The main clinicopathologic variables are shown in Table I. Tumor stage was determined according to Barcelona Clinic Liver Cancer (BCLC) staging classification. Tumor differentiation was graded by the Edmondson grading system. Among these patients, 23 matched fresh HCC tissues and adjacent non-tumor tissues were collected for qRT-PCR and western blot analysis. Written informed consent was obtained from all the patients. None of the patients received any chemotherapy or radiotherapy before surgery. The study was approved by the Ethics Committee of the Second Xiangya Hospital of Central South University.

Follow-up. The follow-up was conducted by telephone or outpatient visits regularly, every 3 months in the first three years after operation and twice a year later. Recurrence or metastasis were monitored by clinical examination, alpha-fetoprotein (AFP) levels and ultrasonography, sometimes high-resolution contrast-enhanced computed tomography (CT) or magnetic resonance imaging (MRI) if necessary. Overall survival (OS) was calculated from the date of surgery to the date of death or the last follow-up. Time to recurrence(TTR) was calculated from the date of surgery to the date of diagnosis of any type of relapse (26). Patient follow-up was terminated on November 10 , 2015. The median follow-up time was 23.5 months, ranging from 1 to 68 months.

RNA isolation and quantitative real-time PCR analysis. TRIzol reagents (Invitrogen, Carlsbad, CA, USA) was used for isolating total RNA from cell lines or tissues. cDNA was generated and quantitative real-time PCR was performed using a standard protocol from the SYBR Green PCR kit (Toyobo, Osaka, Japan). Each sample was analyzed in triplicate. The Primers of ABI1 were as follows: 5'-CGAATAT GGAGCGCCCTGTA-3' (forward); 5'-AGGACTTGGCGGT TTCTGAGT-3' (reverse). GAPDH was used as an internal control with the following primers: 5'-CTGGTAAAGTGGAT ATTGTTGCCAT-3' (forward); 5'-TGGAATCATATTGGAA CATGTAAACC- 3 ' (reverse). The $2^{-\triangle \Delta C t}$ method was used to analyze the qRT-PCR results.

Western blot analysis. Total protein was extracted using RIPA lysis buffer supplemented with $1 \%$ Phenylmethanesulfonyl (PMSF). After separated by SDS-PAGE, the protein was transferred onto PVDF membranes. Then the membranes were blocked with 5\% skimmed milk followed by an overnight incubation with primary antibodies against ABI1 (1:100, Santa Cruz Biotechnology, Santa Cruz, CA, USA) and $\beta$-actin (1:1000, Sigma, St. Louis, MO, USA) at $4^{\circ} \mathrm{C}$. After washing, membranes were incubated with appropriate secondary antibodies conjugated to HRP (1:10000, Zhongshan Goldenbridge Biotechnology, Beijing, China). Signals were detected using the ECL detection systems (Thermo Scientific, Rockford, IL, USA).

Immunohistochemistry (IHC). Tissue sections $(4 \mu \mathrm{m})$ from 124 HCC samples were deparaffinized in xylene and rehydrated
Table I. Correlations between ABI1 expression and clinicopathologic characteristics of 124 HCC patients.

\begin{tabular}{|c|c|c|c|}
\hline \multirow{2}{*}{$\begin{array}{l}\text { Clinicopathological } \\
\text { variables }\end{array}$} & \multicolumn{2}{|c|}{ Tumor ABI1 expression } & \multirow[b]{2}{*}{ P-value } \\
\hline & Low (49) & High (75) & \\
\hline Gender & & & 0.967 \\
\hline Male & 43 & 66 & \\
\hline Female & 6 & 9 & \\
\hline Age & & & 0.752 \\
\hline$<50$ & 28 & 45 & \\
\hline$\geq 50$ & 21 & 30 & \\
\hline AFP & & & 0.146 \\
\hline$<20 \mathrm{ng} / \mathrm{ml}$ & 11 & 26 & \\
\hline$\geq 20 \mathrm{ng} / \mathrm{ml}$ & 38 & 49 & \\
\hline HBsAg & & & 0.640 \\
\hline Negative & 10 & 18 & \\
\hline Positive & 39 & 57 & \\
\hline Liver cirrhosis & & & 0.998 \\
\hline Absent & 17 & 26 & \\
\hline Present & 32 & 49 & \\
\hline Tumor size & & & 0.041 \\
\hline$\leq 5 \mathrm{~cm}$ & 21 & 19 & \\
\hline$>5 \mathrm{~cm}$ & 28 & 56 & \\
\hline Tumor number & & & $<0.001$ \\
\hline Single & 32 & 23 & \\
\hline Multiple & 17 & 52 & \\
\hline Tumor encapsulation & & & $<0.001$ \\
\hline Absent & 25 & 62 & \\
\hline Present & 24 & 13 & \\
\hline Edmondson grade & & & 0.199 \\
\hline I-II & 28 & 34 & \\
\hline III-IV & 21 & 41 & \\
\hline Microvascular invasion & & & 0.101 \\
\hline Absent & 34 & 41 & \\
\hline Present & 15 & 34 & \\
\hline \multicolumn{4}{|l|}{ BCLC stage } \\
\hline $0-\mathrm{A}$ & 16 & 10 & 0.010 \\
\hline B-C & 33 & 65 & \\
\hline
\end{tabular}

HBsAg, hepatitis B surface antigen; AFP, $\alpha$-fetoprotein; BCLC, Barcelona Clinic Liver Cancer. Bold, $\mathrm{P}<0.05$.

with graded ethanol, then antigen retrieval with $0.01 \mathrm{M}$ sodium citrate buffer ( $\mathrm{pH}$ 6.0). The endogenous peroxidase was inactivated with $0.3 \%$ hydrogen peroxide, next with $10 \%$ goat serum blocking for $30 \mathrm{~min}$. ABI1 antibody (1:50, Santa Cruz Biotechnology) was incubated overnight at $4^{\circ} \mathrm{C}$ in a humidified chamber and negative control slides were incubated with PBS. Then followed by HRP conjugated secondary antibody (Zhongshan Goldenbridge Biotechnology) incubation for 
Table II. Univariate and multivariate analysis of prognostic factors associated with OS in 124 HCC patients.

\begin{tabular}{|c|c|c|c|c|c|c|}
\hline \multirow[b]{3}{*}{ Clinicopathological variables } & \multicolumn{6}{|c|}{ OS } \\
\hline & \multicolumn{3}{|c|}{ Univariate analysis } & \multicolumn{3}{|c|}{ Multivariate analysis } \\
\hline & HR & $95 \% \mathrm{CI}$ & P-value & HR & $95 \% \mathrm{CI}$ & P-value \\
\hline Gender (male vs. female) & 0.912 & $0.456-1.826$ & 0.796 & & & NA \\
\hline Age $(<50$ vs. $\geq 50)$ & 0.761 & $0.482-1.200$ & 0.240 & & & NA \\
\hline AFP (<20 vs. $\geq 20$ ng/ml) & 0.775 & $0.482-1.244$ & 0.291 & & & NA \\
\hline HBsAg (negative vs. positive) & 1.390 & $0.793-2.438$ & 0.250 & & & NA \\
\hline Liver cirrhosis (absent vs. present) & 0.807 & $0.511-1.274$ & 0.357 & & & NA \\
\hline Tumor number (single vs. multiple) & 2.112 & $1.329-3.358$ & 0.002 & 1.721 & $1.060-2.793$ & 0.028 \\
\hline Tumor size ( $\leq 5$ vs. $>5 \mathrm{~cm})$ & 1.609 & $0.982-2.635$ & 0.059 & & & NA \\
\hline Tumor encapsulation (present vs. absent) & 1.570 & $0.927-2.660$ & 0.093 & & & NA \\
\hline Tumor differentiation (I-II versus III-IV) & 1.650 & $1.060-2.567$ & 0.026 & & & NS \\
\hline Microvascular invasion (absent vs. present) & 2.098 & $1.349-3.264$ & 0.001 & 1.906 & $1.214-2.993$ & 0.005 \\
\hline BCLC stage (0-A vs. B-C) & 1.897 & $1.026-3.508$ & 0.041 & 2.058 & $1.103-3.841$ & 0.023 \\
\hline ABI1 expression (low vs. high) & 2.327 & $1.428-3.792$ & 0.001 & 1.795 & $1.077-2.990$ & 0.025 \\
\hline
\end{tabular}

HBsAg, hepatitis B surface antigen; AFP, $\alpha$-fetoprotein; BCLC, Barcelona Clinic Liver Cancer; NA, not applicable; NS, not significant.

30 min at room temperature. Antibody binding was detected by DAB. Tissue sections were dehydrated in graded ethanols and mounted.

All the immunostained sections were evaluated by two investigators in a blinded manner. Both the staining intensity and percentage were assessed. Staining intensity was graded on a 0 to 3 scale: 0 (absence of staining), 1 (weakly stained), 2 (moderately stained), and 3 (strongly stained). The percent positivity was scored as $1(0-25 \%), 2(26-50 \%), 3(51-75 \%)$ and $4(\geq 75 \%)$. A final score was obtained for each case by multiplying the percentage and the intensity score. Therefore, tumors with a multiplied score exceeding 4 were deemed to be high ABI1 expression; all other scores were considered to be low ABI1 expression.

Cell culture. The human HCC cell lines (Hep3B, HepG2, MHCC97H and SMMC7721) and normal liver cell line L02 were purchased from the Shanghai Institute of Cell Biology. The cells were maintained in Dulbecco's modified Eagle's medium (DMEM) supplemented with $10 \%$ fetal bovine serum(FBS) (Gibco, Australia) and $100 \mathrm{U} / \mathrm{ml}$ penicillinstreptomycin mixture at $37^{\circ} \mathrm{C}$ in $5 \% \mathrm{CO}_{2}$.

Construction of stable cell lines. Human Lenti-ABI1-GFP and three Lenti-shABI1-GFP as well as their negative control lentiviruses were designed and purchased from GenePharma Technologies (Shanghai, China). The transfection was performed according to standard procedures. Following lentiviral infection, single-cell clonal isolates were selected in the presence of puromycin for 2-4 weeks. The 3 candidate hairpin sequences for ABI1 were as follows: 5'-GGAGTCTTCCAT CAATCATAT-3' (shRNA-1); 5'-GGTATATTCGGAAAC CTATCG-3' (shRNA-2); 5'-GCACACTGTCGAGAACAA ATC-3' (shRNA-3); The efficiency of ABI1 overexpression or knockdown was confirmed by qRT-PCR and western blotting after transfection, respectively.

Cell Counting Kit-8 assay and Colony formation assay. Cell proliferation was determined by the Cell Counting Kit- 8 method (Dojindo Laboratories, Kumamoto, Japan) according to the manufacturer's instructions. The cell proliferation curves were plotted using the absorbance at each time point. Experiments were performed in triplicate.

For colony formation assay, $5 \times 10^{2}$ cells were seeded in each well on 6-well plates and incubation for 14 days, then cells were washed twice with PBS, fixed with methanol and stained with crystal violet. The number of colonies $>40 \mu \mathrm{m}$ in diameter was counted after the dishes were captured with a camera.

EdU incorporation assay. EdU incorporation assay was carried out using the Cell-Light EdU imaging kit (RiboBio, Guangzhou, China) according to the manufacturer's protocol. Briefly, $5 \times 10^{3}$ cells were seeded in each well on 96-well plates. After adherence, we added EdU labeling medium and incubated for approximately $60 \mathrm{~min}$. Then cells were fixed with $4 \%$ formaldehyde for $15 \mathrm{~min}$ and successively treated with $0.5 \%$ Triton X-100 for $10 \mathrm{~min}$, Apollo reaction cocktail for $30 \mathrm{~min}$ and Hoechst $33342(5 \mu \mathrm{g} / \mathrm{ml})$ for $30 \mathrm{~min}$. The results were visualized under a florescent microscope. For quantification of HCC cell proliferative rate, five randomly selected views from each sample image were used to calculate the relative EdU-positive ratio.

Wound healing assay. Cells $\left(1 \times 10^{5}\right)$ were seeded in each well on 6-well plates and when the cell confluence reached approximately $90 \%$, a line was scraped using the fine end of a $10 \mu \mathrm{l}$ pipette tip. Serum-free medium with Mitomycin-C $(10 \mu \mathrm{g} / \mathrm{ml})$ was used to suppress cell proliferation (27). Wound healing 
within the scrape line was observed and photographed every $12 \mathrm{~h}$. Each experiment was repeated three times.

Transwell invasion assay. For the cell invasion assay, Transwell chambers (Corning, 8- $\mu \mathrm{m}$ pore size) were coated with $200 \mu \mathrm{l}$ Matrigel at $200 \mu \mathrm{g} / \mathrm{ml}$ and incubated overnight. Cells $\left(1 \times 10^{5}\right)$ were suspended in serum-free DMEM and plated into the upper chamber. The lower chamber was filled with DMEM containing $10 \%$ FBS. After a $48 \mathrm{~h}$ incubation in $5 \% \mathrm{CO}_{2}$ at $37^{\circ} \mathrm{C}$, the cells in the upper chamber were removed and the bottom surface of the polycarbonate membranes was stained using $0.1 \%$ crystal violet dye. Cell invasion was determined by counting six random fields under a microscope. All assays were carried out in triplicates.

Immunofluorescence (IF). For immunofluorescence of cytoskeleton, cells were fixed in $4 \%$ paraformaldehyde, permeabilized using $0.5 \%$ Triton X-100 and incubated with Phalloidin (Sigma) according to the manufacturer's protocol. The coverslips were counterstained with DAPI and imaged with an inverted microscope.

Subcutaneous and lung metastasis tumor models in nude mice. All the animal experiments were performed in accordance with the guidelines of the Laboratory Animal Ethics Committee of Central South University. For the in vivo tumorigenesis model, cells $\left(5 \times 10^{6}\right)$ resuspended in $100 \mu$ of PBS were injected subcutaneously to the left side of nude mice (4 mice/per group). Four weeks later, the subcutaneous tumors were resected, and tumor size was monitored by digital caliper and tumor volume was calculated with the following formula: $1 / 2$ length $\mathrm{x}$ width ${ }^{2}$.

For the in vivo lung metastasis model, cell suspension at a concentration of $1 \times 10^{7}$ cells ml ${ }^{-1}$ was injected into nude mice through tail veins (4 mice/per group). Six weeks later, the mice were sacrificed, and the numbers of lung metastatic nodules were carefully examined and counted under a microscope.

Statistical analysis. Statistical analysis was performed using SPSS18.0 (IBM, Chicago, IL, USA). All quantified data are presented as the mean \pm SD. Two-tailed Student's t-test was used for comparisons of two independent groups. The association between ABI1 and clinicopathological features were analyzed using the Chi-square method. Kaplan-Meier curves were constructed, and the log-rank test was used for analyzing the survival data. Univariate and multivariate analyses were based on the Cox proportional hazards regression model. $\mathrm{P}<0.05$ was set as the statistical significance.

\section{Results}

ABII is significantly upregulated in human HCC tissues. To clarify the underlying role of ABI1 in HCC progression, we first retrieved the expression of ABI1 from Oncomine Database (www.oncomine.org). Data showed that the level of ABI1 mRNA was significantly upregulated in HCC tissues relative to normal liver tissues in both Roessler liver 1 and 2 dataset (Fig. 1A1 and A2). Convincingly, similar results were also observed in Chen liver dataset, Mas liver dataset and Wurmbach liver dataset, while P-values were 1.31E-5,
1.120E-5 and 5.10E-5, respectively (Fig. 1A3-A5). To further confirm the above findings, quantitative real-time PCR (qRT-PCR) and western blot were performed to detect the expression of ABI1 in 23 fresh-frozen HCC samples. As shown in Fig. 1B and C, both the mRNA and protein levels of ABI1 were significantly higher in HCC tissues compared with the matched non-tumor tissues $(\mathrm{P}<0.001)$. Furthermore, we performed immunohistochemistry (IHC) to examine the expression of ABI1 protein in 124 paraffin-embedded HCC samples. Representative images of ABI1 staining are shown in Fig. 1D. The positive signal for ABI1 was observed primarily in the cytoplasm of hepatic cells. Of the $124 \mathrm{HCC}$ specimens, 75 (60.5\%) displayed high ABI1 expression and 49 (39.5\%) had low ABI1 expression. However, in adjacent non-tumor tissues, only 37 (29.8\%) exhibited high ABI1 expression. The expression levels of ABI1 were significantly increased in HCC tissues relative to matched non-tumor tissues $(\mathrm{P}<0.001$; Fig. 1E). Taken together, the above results suggest that ABI1 is significantly upregulated in HCC tissues and may play an important role in HCC development.

High ABIl expression associates with malignant clinicopathological features and predicts poor prognosis in $\mathrm{HCC}$ patients. To illustrate the clinical significance of ABI1 expression in HCC, we first analyzed the correlation between ABI1 expression and clinicopathological features of HCC patients. As shown in Table I, high ABI1 expression was significantly correlated with tumor size $(\mathrm{P}=0.041)$, tumor number $(\mathrm{P}<0.001)$, tumor encapsulation $(\mathrm{P}<0.001)$, and $\mathrm{BCLC}$ stage $(\mathrm{P}=0.010)$, but did not correlate with other clinicopathologic characteristics including age, gender, serum AFP level, HBsAg, liver cirrhosis, tumor differentiation and microvascular invasion. Importantly, Kaplan-Meier analysis revealed that patients with high ABI1 expression had shorter overall survival time (median OS 25.7 vs. 40.2 months, $\mathrm{P}<0.001$ ) and a higher tendency of tumor recurrence (median TTR 23.4 vs. 37.0 months, $\mathrm{P}=0.001$ ) than those with low ABI1 expression (Fig. 2A and B). Strikingly, as shown in Tables II and III, multivariate analysis further confirmed that high ABI1 expression was an independent predictor for both OS (hazard ratio 1.795, 95\% confidence interval 1.077-2.990, $\mathrm{P}=0.025$ ) and TTR (hazard ratio 1.893, $95 \%$ confidence interval 1.152-3.109, $\mathrm{P}=0.012$ ). These results indicate that $\mathrm{ABI} 1$ may be a very promising prognostic indicator for patients with HCC.

Construction of stable cell lines displaying ABII overexpression or knockdown. We next analyzed the expression of ABI1 in four different human HCC cell lines (HepG2, Hep3B, SMMC7721 and MHCC97H) and immortalized hepatocytes (L02). Results showed that both levels of ABI1 mRNA and protein in HCC cell lines were evidently higher than those in L02 (Fig. 3A and B). Noteworthy, we found that the highly metastatic cell line (MHCC97H and Hep3B) exhibited stronger signals of ABI1 than the lowly metastatic cell line (HepG2 and SMMC7721). To further elucidate the biological function of ABI1 in HCC cells, we chose HepG2 and MHCC97H cells for further research, which expressed the lowest and highest levels of ABI1 among the four examined cell lines, respectively. Then we stably overexpressed ABI1 in HepG2 and silenced ABI1 in MHCC97H cells by 

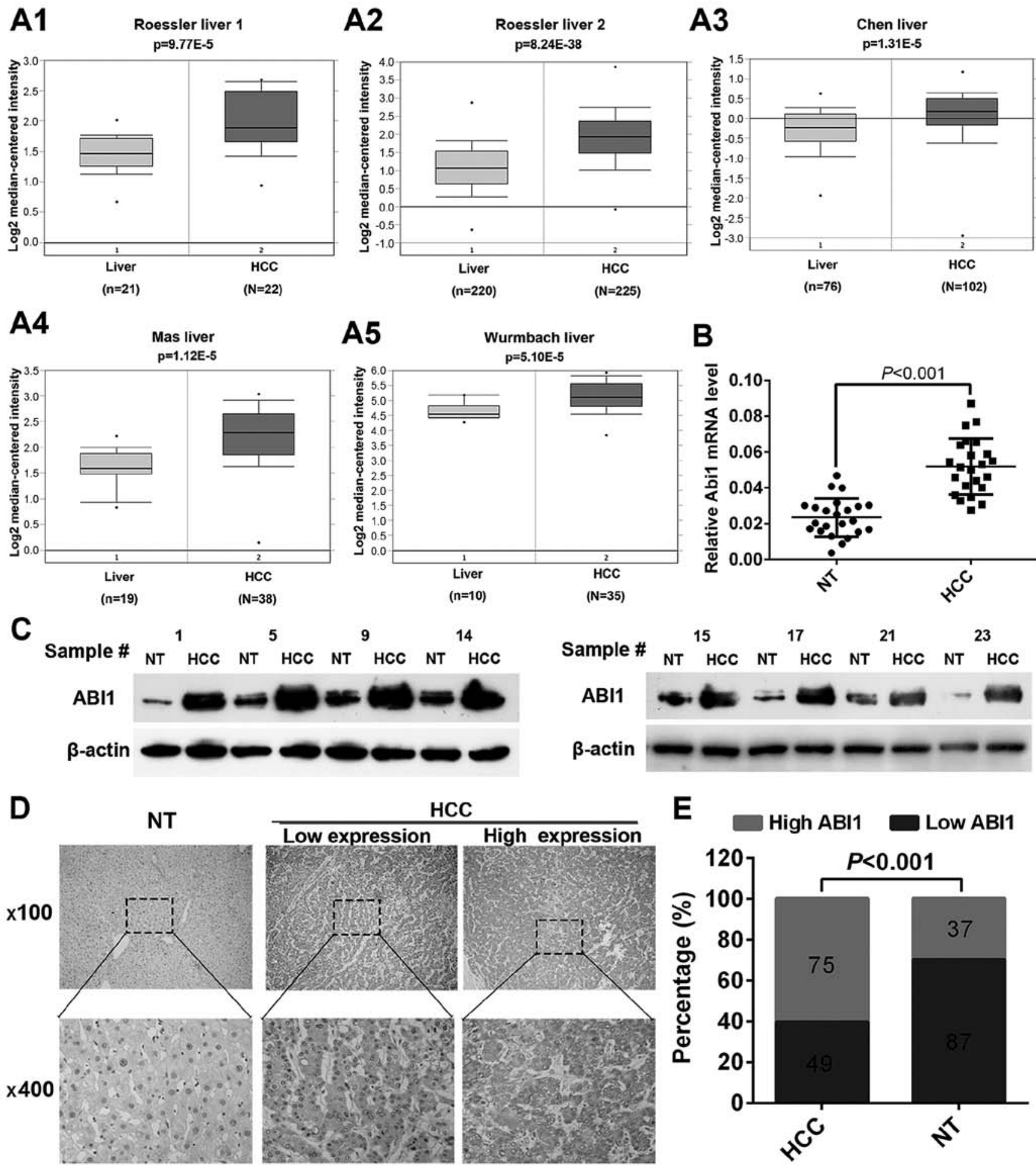

Figure 1. ABI1 is significantly upregulated in human HCC tissues. (A1-5) The mRNA level of ABI1 is significantly increased in five cohorts of HCC patients, (detailed information in Oncomine database) by Roessler in 2010 (A1-2), Chen in 2002 (A3), Mas in 2008 (A4) and Wurmbach in 2007 (A5), respectively. (B) The relative expression level of ABI1 mRNA in 23 paired HCC tissues and matched non-tumor tissues (NTs). (C) Representative western blot results of the amounts of ABI1 in HCC tissues and NTs. $\beta$-actin was used as the loading control. (D) ABI1 protein expression was examined by immunohistochemical analysis in $124 \mathrm{HCC}$ samples and the representative results are shown. (E) The numbers and percentages of high and low expression of ABI1 in HCC tissues and NTs.

lentivirus transfection. After transfection, qRT-PCR and western blot were used to evaluate the efficiency of ABI1 overexpression or knockdown in HCC cells. Results showed that transfection of ABI1 expressing lentiviral plasmid increased the expression of ABI1 in HepG2 cells (Fig. 3C and D). Among the three shRNAs, ABI1 was significantly knocked down by shRNA-2 (Fig. 3E and F), which was chosen for further study.

ABII promotes $H C C$ cell growth in vitro and in vivo. To evaluate the function of ABI1 on cell proliferation in HCC, Cell Counting Kit-8 assay, clone formation and EdU assays 

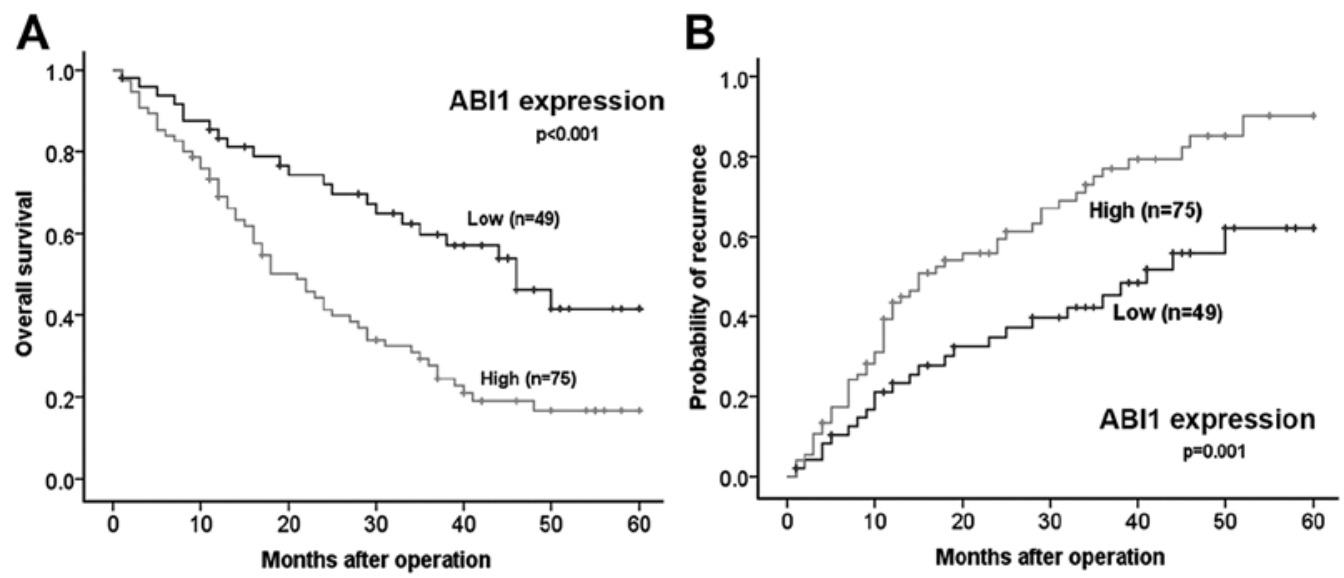

Figure 2. Prognostic significance of ABI1 was assessed by Kaplan-Meier analysis and log-rank tests. HCC patients with high ABI1 expression presented with poor overall survival (A) and high cumulative recurrence rates (B).

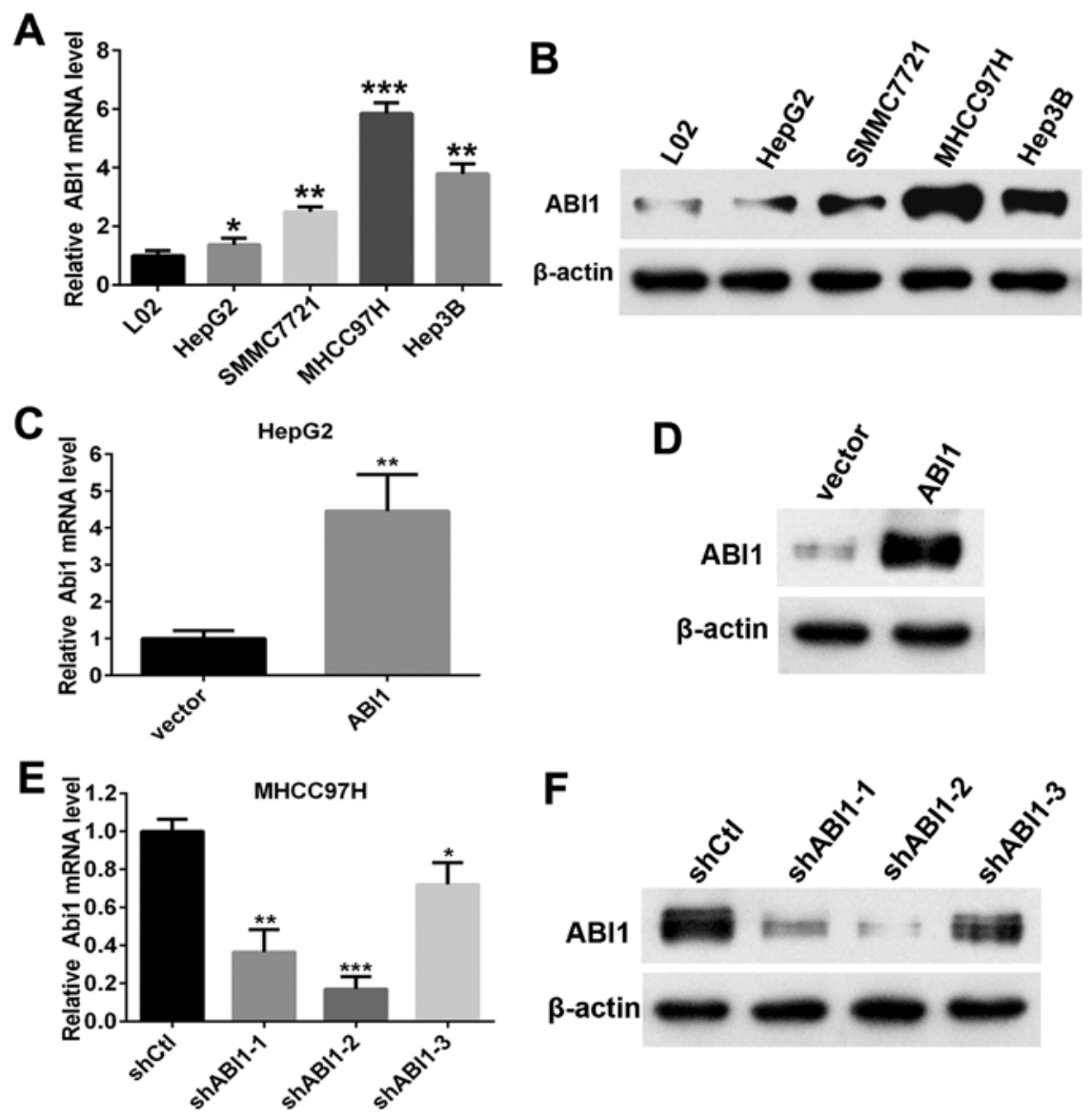

Figure 3. Expression and transfection efficiency of ABI1 in HCC cell lines. (A and B) qRT-PCR and western blot analysis of ABI1 expression in the normal liver cell line L02 and 4 HCC cell lines (HepG2, SMMC7721, MHCC97H and Hep3B). (C and D) qRT-PCR and western blot analysis showed that HepG2 cells stably overexpressed ABI1 after infected with lentivirus carrying the ABI1 gene. (E and F) shRNA against ABI1 stably decreased the expression of ABI1 in $\mathrm{MHCC} 97 \mathrm{H}^{\text {shABII }}$ cells compared with $\mathrm{MHCC} 97 \mathrm{H}^{\text {shCll }}$ cells by qRT-PCR and western blotting. All results are from three independent experiments. "P<0.05, ${ }^{* * *} \mathrm{P}<0.01,{ }^{* * *} \mathrm{P}<0.001$.

in vitro were performed. The results of CCK8 assay and clone formation assay showed that overexpression of ABI1 in HepG2 cells significantly promoted cell viability and colony formation compared with HepG2 $2^{\text {vector }}$ cells, whereas knockdown of ABI1 in MHCC97H cells had the opposite effect (Fig. 4A and B). In addition, similar results were observed in EdU incorporation assays (Fig. 4C).
To further verify the growth-enhancing effect of ABI1 in vivo, $\mathrm{HepG} 2^{\mathrm{ABII}}$, MHCC $97 \mathrm{H}^{\text {shABII }}$ and their control cells were injected subcutaneously into nude mice for xenotransplantation. Data showed that ABI1 overexpression in HepG2 cells resulted in a marked increase in tumor size compared with the control transfectants. Conversely, ABI1 knockdown in MHCC97H cells decreased the tumor size (Fig. 4D). These 
A
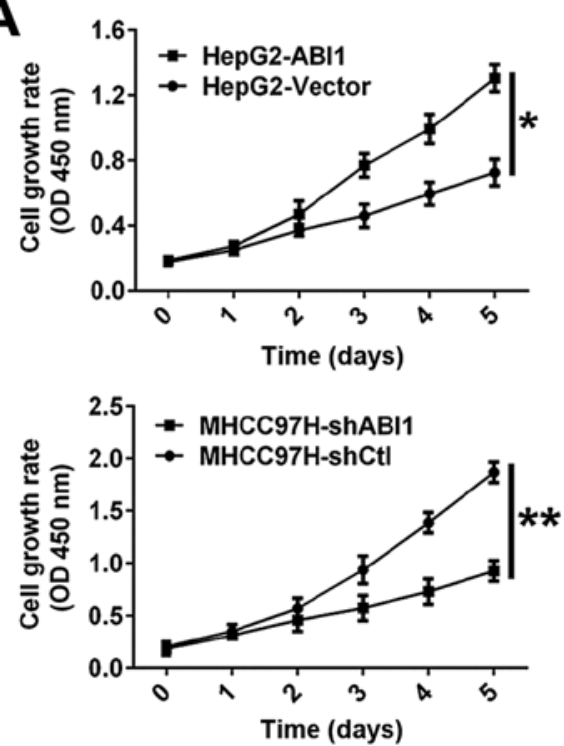
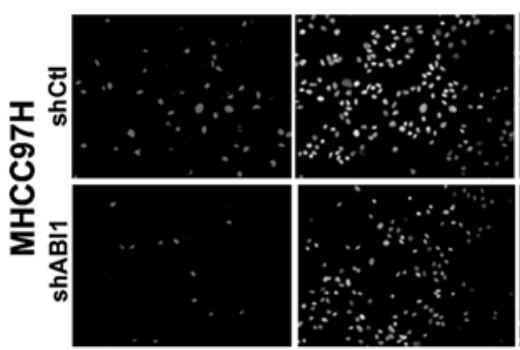

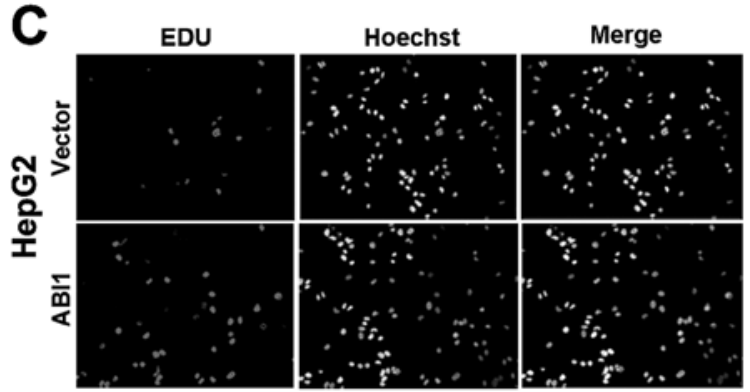

B
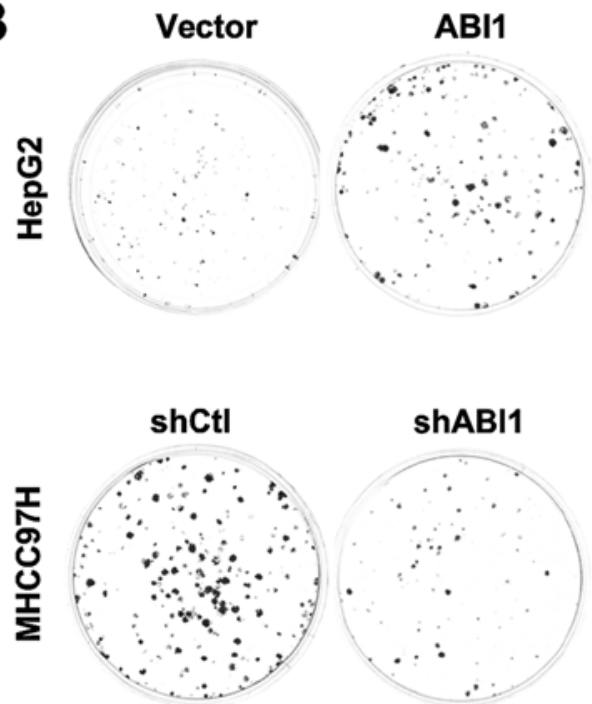
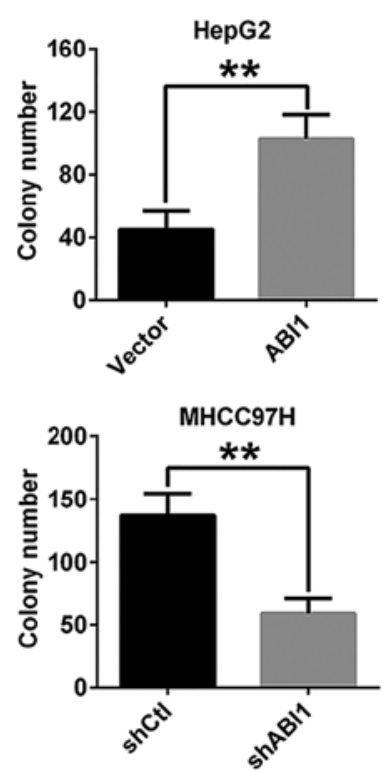

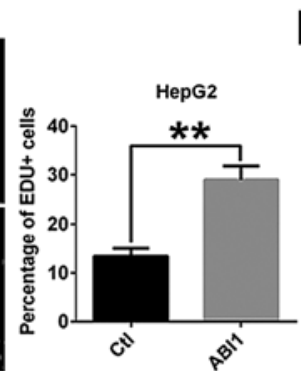

D
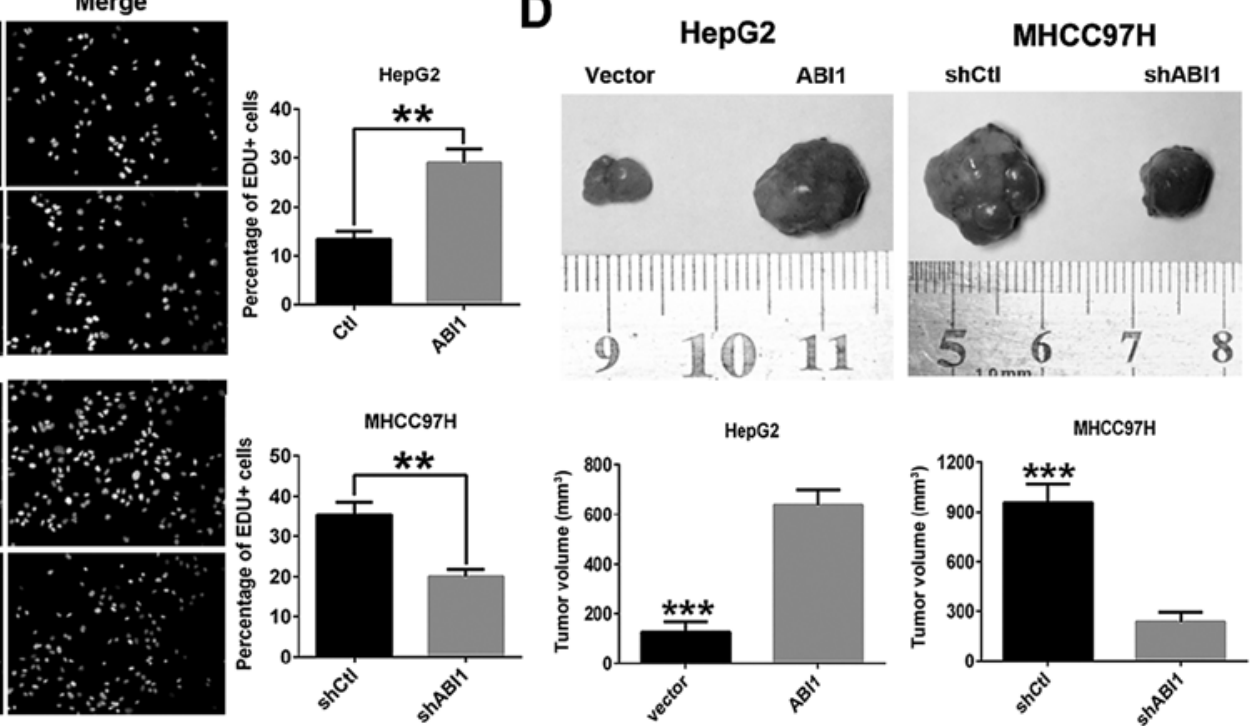

Figure 4. ABI1 promotes proliferation of HCC cells both in vitro and in vivo. (A) CKK-8 analysis of the proliferation ability of HCC cells transfected with ABI1, shABI1 or control. (B) Representative micrographs (left) and quantification (right) of HCC cell colonies determined by colony formation assay. (C) EdU staining patterns (left) and the relative EdU-positive cells ratio (right) of the indicated HCC cell lines. Magnification, x200. (D) Representative images (upper) and size (lower) of the xenograft tumor in different groups. ${ }^{*} \mathrm{P}<0.05,{ }^{* * *} \mathrm{P}<0.01,{ }^{* * * *} \mathrm{P}<0.001$.

results provided evidence that ABI 1 accelerates HCC cell proliferation both in vitro and in vivo.

ABII enhances HCC cell migration, invasion and lung metastasis. In order to investigate the effects of ABI1 on HCC cell motility, wound healing and Transwell invasion assays were performed. The results showed that HepG2 $2^{\mathrm{ABI}}$ cells had significantly higher percentage of wound closure compared with the control cells (Fig. 5A). Moreover, HepG2ABI1 cells also showed a high degree of invasion through Matrigel (Fig. 5B). By contrast, silencing ABI1 markedly decreased the percentage of wound closure and number of invasion cells in MHCC97H (Fig. 5A and B). Since previous studies showed that ABI1 plays an important role in the cell cytoskeleton rearrangements $(28,29)$, which is crucial for the invasive and metastatic spread of tumor cells, we performed rhodamine-phalloidin fluorescent staining to examine morphologic changes of HCC cells. As shown in Fig. 5C, ectopic expression of ABI1 in HepG2 cells displayed fibroblast-like spindle shape with long stretched F-actin fibers. However, silenced ABI1 in MHCC97H cells exhibited cobblestone-like appearance with shrunken F-actin fibers.

To verify these findings in vivo, we generated lung metastasis model in nude mice by injecting HepG2 $2^{\mathrm{ABI}}$, MHCC $97 \mathrm{H}^{\text {shABI1 }}$ and their parental control cells intravenously in the tail vein. Six weeks later, the mice were sacrificed and the metastatic nodules in the lungs were counted. The representative images of lungs with/without metastasis are shown in Fig. 5D. Hematoxylin and eosin (H\&E) staining confirmed that the nodules in the lungs were metastatic tumors (Fig. 5E). Further analysis showed that nude mice inoculated with HepG2 ${ }^{\mathrm{ABI1}}$ cells displayed more micrometastatic lesions in 
A

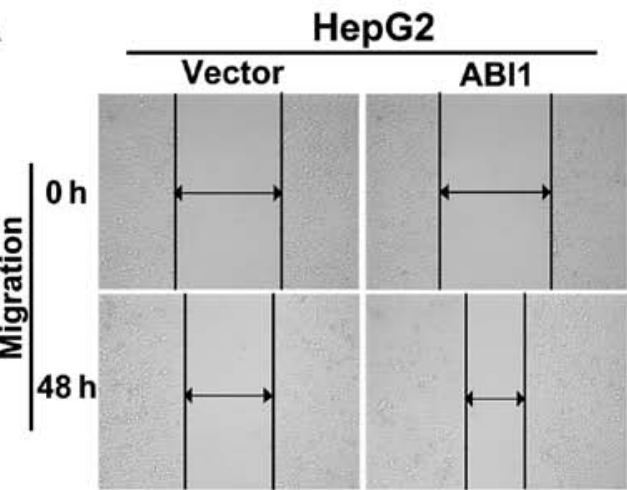

B
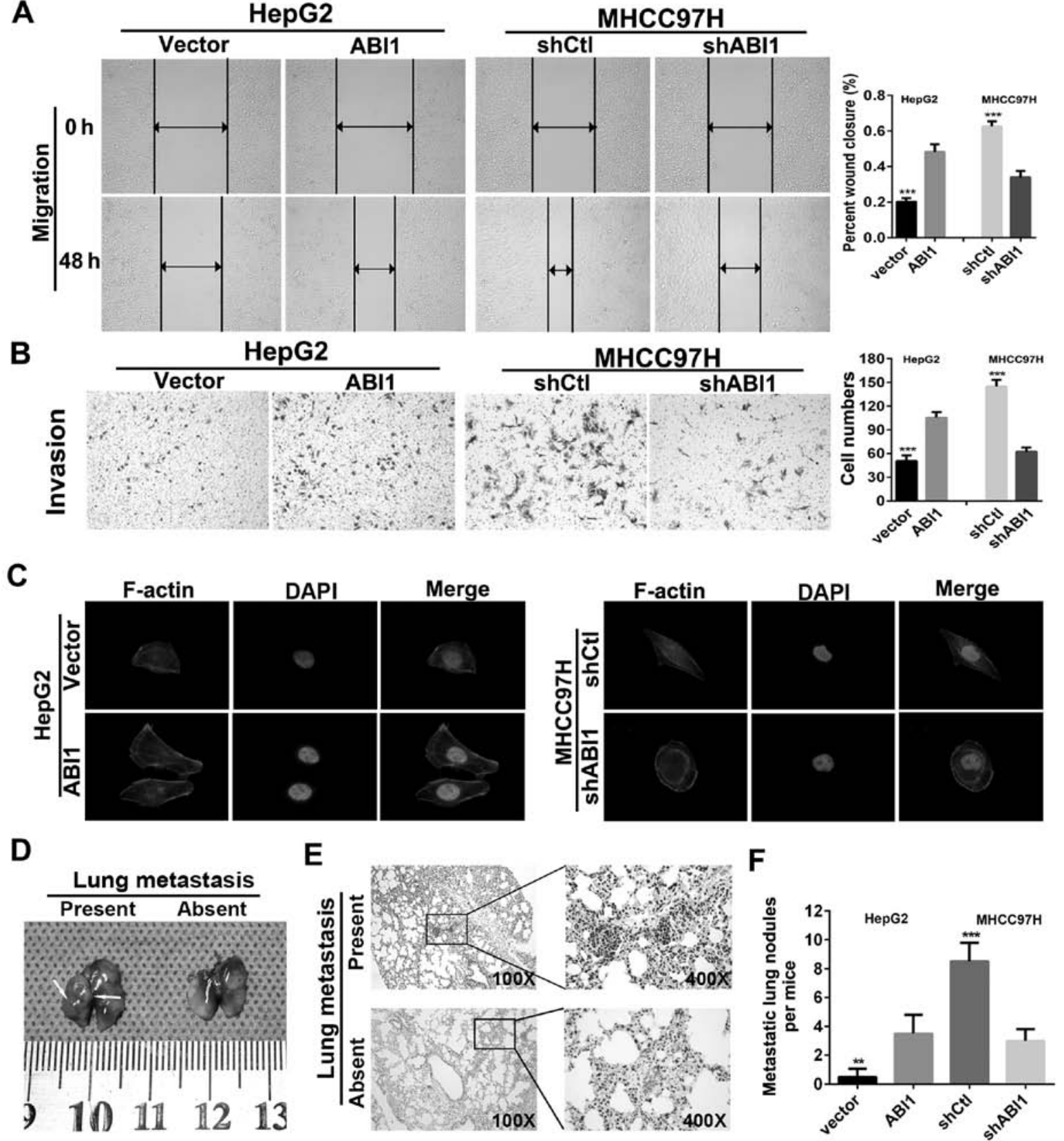

Figure 5. ABI1 enhances HCC cell migration, invasion and lung metastasis. (A) The migration property of HCC was assessed by wound healing assay. Representative images (left, x200) and quantitative data (right) of wound healing assay are shown. (B) The invasion properties of HCC cells were determined by Transwell invasion assay. Representative images (left, x200) and quantification of the five randomly selected fields (right) are shown. (C) The effects of ABI1 on cytoskeletal rearrangement in HCC cells. (D) The effects of ABI1 on HCC metastasis in vivo was determined by lung metastasis model in nude mice. Representative images show lungs with/without metastatic HCC tumors. Arrows indicated tumor nodules at the surface of the lungs. (E) Representative images of hematoxylin and eosin (H\&E) sections derived from lung tissues with/without metastasis (left: magnification, x100; right: magnification, $x$ 400). (F) Numbers of metastatic nodules in the lungs from the ABI1-interfered HCC cells are shown. The are data shown as the mean \pm SD of three independent experiments. ${ }^{* *} \mathrm{P}<0.01,{ }^{* * *} \mathrm{P}<0.001$.

lungs as compared with HepG2 $2^{\text {vector }}$ cells. However, nude mice in $\mathrm{MHCC} 97 \mathrm{H}^{\text {shABI1 }}$ groups exhibited less micrometastatic lesions than $\mathrm{MHCC} 97 \mathrm{H}^{\text {shCtl }}$ groups (Fig. 5F). Hence, these observations suggest that ABI1 is crucial for promoting HCC invasion and metastasis.

\section{Discussion}

The major obstacle for improving the outcome of cancer patients is recurrence and metastasis. Given that actin polymerization and lamellipodia formation are critical steps in cell migration during metastasis (30), while ABI1 was initially reported to exert its regulatory role in actin reorganization and lamellipodia formation, the function of ABI1 in cancer development have attracted increased attention. Accumulating evidence has indicated that ABI1 was frequently dysregulated in the development of cancer, however, it remains controversial whether ABI1 acts as a tumor promoter or suppressor. On the one hand, some studies reported that ABI1 overexpression was closely associated with tumor progression in breast cancer (20), colon 
Table III. Univariate and multivariate analysis of prognostic factors associated with TTR in 124 HCC patients.

\begin{tabular}{|c|c|c|c|c|c|c|}
\hline \multirow[b]{3}{*}{ Clinicopathological variables } & \multicolumn{6}{|c|}{ TTR } \\
\hline & \multicolumn{3}{|c|}{ Univariate analysis } & \multicolumn{3}{|c|}{ Multivariate analysis } \\
\hline & HR & $95 \% \mathrm{CI}$ & P-value & HR & $95 \% \mathrm{CI}$ & P-value \\
\hline Gender (male vs. female) & 0.793 & $0.369-1.590$ & 0.514 & & & NA \\
\hline Age (<50 vs. $\geq 50)$ & 0.850 & $0.540-1.335$ & 0.480 & & & NA \\
\hline AFP (<20 vs. $\geq 20 \mathrm{ng} / \mathrm{ml})$ & 0.810 & $0.501-1.311$ & 0.391 & & & NA \\
\hline HBsAg (negative vs. positive) & 1.725 & $0.950-3.130$ & 0.073 & & & NA \\
\hline Liver cirrhosis (absent vs. present) & 0.774 & $0.491-1.219$ & 0.269 & & & NA \\
\hline Tumor number (single vs. multiple) & 1.937 & $1.223-3.070$ & 0.005 & & & NS \\
\hline Tumor size ( $\leq 5$ vs. $>5$ ) & 1.351 & $0.839-2.174$ & 0.216 & & & NA \\
\hline Tumor encapsulation (present vs. absent) & 1.310 & $0.793-2.165$ & 0.292 & & & NA \\
\hline Tumor differentiation (I-II vs. III- IV) & 1.770 & $1.130-2.772$ & 0.013 & 1.629 & $1.035-2.567$ & 0.035 \\
\hline Microvascular invasion (absent vs. present) & 2.190 & $1.401-3.423$ & 0.001 & 2.050 & $1.298-3.237$ & 0.002 \\
\hline BCLC stage (0-A vs. B-C) & 1.947 & $1.051-3.605$ & 0.034 & 1.982 & $1.054-3.730$ & 0.034 \\
\hline ABI1 expression (low vs. high) & 2.142 & $1.318-3.479$ & 0.002 & 1.893 & $1.152-3.109$ & 0.012 \\
\hline
\end{tabular}

HBsAg, hepatitis B surface antigen; AFP, $\alpha$-fetoprotein; BCLC, Barcelona Clinic Liver Cancer; NA, not applicable; NS, not significant.

cancer $(22,23)$, HCC (25) and ovarian cancer (24). which revealed a potential carcinogenic role of $\mathrm{ABI} 1$ in cancers. However, some other studies showed that ABI1 expression was decreased in human prostate cancer, gastric cancer and some types of leukemia (14-17) compared to corresponding normal tissues. These contradictory results indicated that the ABI1 function as oncogenes or tumor suppressor genes was context dependent. However, there are limited studies on the expression, significance, and function of ABI1 in HCC.

In this study, we for the first time evaluated the ABI1 expression and clinical significance in a large cohort of HCC patients. Before the experiments started, we first collected ABI1 information through bioinfomatic analysis from Oncomine database and found that the levels of ABI1 mRNA were significantly increased in five independent liver cancer datasets. Next, our results in 23 paired HCC samples further confirmed the upregulation of ABI1 in HCC tissues. When evaluated the level of ABI1 protein by IHC in an expanded population with 124 pairs of HCC samples, we also observed that the ABI1 protein was increased in $60.5 \%$ of paraffinembedded HCC tissues, which was predominantly detected in the cytoplasm of HCC cells. Our findings are consistent with the result from Liu et al in $40 \mathrm{HCC}$ samples (25) but more convincing. Collectively, our results unambiguously confirmed that ABI1 is overexpressed in HCC tissues, which may be an oncogene in HCC.

Moreover, several pieces of evidence in this study support a close association between ABI1 expression and HCC progression. Firstly, we provided evidence that increased expression of ABI1 was significantly associated with invasive characteristics of $\mathrm{HCC}$, including multiple tumor nodes, larger tumor size, lack of tumor encapsulation and advanced BCLC stage. Secondly, survival analysis demonstrated that HCC patients with high ABI1 expression had significantly shorter
OS time and TTR than did patients with low ABI1 expression. Moreover, multivariate analyses disclosed that ABI1 expression level was an independent risk factor for poor survival of HCC patients after curative resection. These clinical data strongly suggest that ABI1 contributes to the malignant progression of HCC, thus making it a useful prognostic biomarker. Actually, our results are consistent with previous studies in other cancers. Wang et al (20) performed IHC in a tissue microarrays including 988 invasive breast carcinoma patients and found that tumors expressing high levels of ABI1 were significantly associated with early recurrence and worse survival. Steinestel et al $(22,23)$ reported ABI1 overexpression in primary human colorectal carcinoma associated with an infiltrative phenotype and high-grade tumor cell budding. Zhang et al (24) observed that upregulation of ABI1 predicted poor outcome in epithelial ovarian cancer.

Although previous studies found that overexpression of ABI1 promotes cell proliferation, migration, and invasion in some cancer types $(18,19,21)$, Kumar et al recently reported that ABI1 negatively regulated Tyr251 phosphorylation of Crk and inhibited invasive behavior of glioblastoma cells (31). Thus, these characteristics of ABI1 are further needed to confirm in HCC cells for detailed phenotypes. Firstly, we determined the expression pattern of ABI1 in HCC cell lines and manipulated the expression of ABI1 in HCC cells by lentivirus transfection. Moreover, we explored the potential roles of ABI1 in tumor cell proliferation, migration and invasion. In our study, we found that ABI1 overexpression significantly promoted HCC cell proliferation, migration and invasion in vitro. Otherwise, ABI1 knockdown prominently reduced them. Furthermore, the mouse model experiments revealed that ABI1 not only promoted HCC growth, but also enhanced metastatic potential. Altogether, dysregulation of ABI1 may play a fundamental role in tumor growth and metastasis. To 
our knowledge, this is the first study exploring the biological function of ABI1 in HCC. However, the potential mechanism by which $\mathrm{ABI} 1$ affects $\mathrm{HCC}$ progression remains elusive and will require future investigation.

In conclusion, our study revealed that ABI1 was frequently upregulated in HCC tissues and significantly associated with disease progression and poor post-operative outcome of HCC patients. Functionally, ABI1 promoted HCC growth and metastasis both in vitro and in vivo. Thus, ABI1 acts as an oncogene in HCC progression and is a novel prognostic molecular marker for patients with HCC.

\section{Acknowledgements}

This study was supported by grants from the Fundamental Research Funds for the Central Universities of Central South University (2014zzts088).

\section{References}

1. Schütte K, Bornschein J and Malfertheiner P: Hepatocellular carcinoma - epidemiological trends and risk factors. Dig Dis 27 80-92, 2009.

2. He J, Gu D, Wu X, Reynolds K, Duan X, Yao C, Wang J, Chen CS, Chen J, Wildman RP, et al: Major causes of death among men and women in China. N Engl J Med 353: 1124-1134, 2005.

3. Forner A, Llovet JM and Bruix J: Hepatocellular carcinoma. Lancet 379: 1245-1255, 2012.

4. Shi Y, Alin K and Goff SP: Abl-interactor-1, a novel SH3 protein binding to the carboxy-terminal portion of the Abl protein suppresses v-abl transforming activity. Genes Dev 9: 2583-2597, 1995.

5. Brehme M, Hantschel O, Colinge J, Kaupe I, Planyavsky M, Köcher T, Mechtler K, Bennett KL and Superti-Furga G: Charting the molecular network of the drug target Bcr-Abl. Proc Natl Acad Sci USA 106: 7414-7419, 2009.

6. Scita G, Nordstrom J, Carbone R, Tenca P, Giardina G, Gutkind S, Bjarnegård M, Betsholtz C and Di Fiore PP: EPS8 and E3B1 transduce signals from Ras to Rac. Nature 401: 290-293, 1999.

7. Scita G, Tenca P, Areces LB, Tocchetti A, Frittoli E, Giardina G, Ponzanelli I, Sini P, Innocenti M and Di Fiore PP: An effector region in Eps8 is responsible for the activation of the Rac-specific GEF activity of Sos-1 and for the proper localization of the Rac-based actin-polymerizing machine. J Cell Biol 154 1031-1044, 2001.

8. Kotula L: Abil, a critical molecule coordinating actin cytoskeleton reorganization with PI-3 kinase and growth signaling. FEBS Lett 586: 2790-2794, 2012.

9. Dubielecka PM, Ladwein KI, Xiong X, Migeotte I, Chorzalska A, Anderson KV, Sawicki JA, Rottner K, Stradal TE and Kotula L: Essential role for Abil in embryonic survival and WAVE2 complex integrity. Proc Natl Acad Sci USA 108: 7022-7027, 2011

10. Steffen A, Rottner K, Ehinger J, Innocenti M, Scita G, Wehland J and Stradal TE: Sra-1 and Nap1 link Rac to actin assembly driving lamellipodia formation. EMBO J 23: 749-759, 2004.

11. Kheir WA, Gevrey JC, Yamaguchi H, Isaac B and Cox D: A WAVE2-Abi1 complex mediates CSF-1-induced F-actin-rich membrane protrusions and migration in macrophages. J Cell Sci 118: 5369-5379, 2005.

12. Chen H, Wu X, Pan ZK and Huang S: Integrity of SOS1/EPS8/ ABI1 tri-complex determines ovarian cancer metastasis. Cancer Res 70: 9979-9990, 2010.

13. Ring $\mathrm{C}$, Ginsberg $\mathrm{MH}$, Haling $\mathrm{J}$ and Pendergast AM: Abl-interactor-1 (Abi1) has a role in cardiovascular and placental development and is a binding partner of the alpha4 integrin. Proc Natl Acad Sci USA 108: 149-154, 2011.
14. Macoska JA, Xu J, Ziemnicka D, Schwab TS, Rubin MA and Kotula L: Loss of expression of human spectrin src homology domain binding protein 1 is associated with $10 \mathrm{p}$ loss in human prostatic adenocarcinoma. Neoplasia 3: 99-104, 2001.

15. Dai Z, Quackenbush RC, Courtney KD, Grove M, Cortez D, Reuther GW and Pendergast AM: Oncogenic Abl and Src tyrosine kinases elicit the ubiquitin-dependent degradation of target proteins through a Ras-independent pathway. Genes Dev 12: 1415-1424, 1998

16. Cui M, Yu W, Dong J, Chen J, Zhang X and Liu Y: Downregulation of ABI1 expression affects the progression and prognosis of human gastric carcinoma. Med Oncol 27: 632-639, 2010.

17. Jenei $\mathrm{V}$ and Jakus $\mathrm{J}$ : The role of EGF receptor-dependent e3B1/Abi1 protein as a tumor suppressor protein in malignant tumors. Orv Hetil 146: 1293-1299, 2005 (In Hungarian).

18. Wang C, Navab R, Iakovlev V, Leng Y, Zhang J, Tsao MS, Siminovitch K, McCready DR and Done SJ: Abelson interactor protein-1 positively regulates breast cancer cell proliferation, migration, and invasion. Mol Cancer Res 5: 1031-1039, 2007.

19. Sun X, Li C, Zhuang C, Gilmore WC, Cobos E, Tao Y and Dai Z: Abl interactor 1 regulates Src-Id1-matrix metalloproteinase 9 axis and is required for invadopodia formation, extracellular matrix degradation and tumor growth of human breast cancer cells. Carcinogenesis 30: 2109-2116, 2009.

20. Wang C, Tran-Thanh D, Moreno JC, Cawthorn TR, Jacks LM, Wang DY, McCready DR and Done SJ: Expression of Abl interactor 1 and its prognostic significance in breast cancer: A tissue-array-based investigation. Breast Cancer Res Treat 129: 373-386, 2011.

21. Yu W, Sun X, Clough N, Cobos E, Tao Y and Dai Z: Abil gene silencing by short hairpin RNA impairs Bcr-Abl-induced cell adhesion and migration in vitro and leukemogenesis in vivo. Carcinogenesis 29: 1717-1724, 2008.

22. Steinestel K, Brüderlein S, Steinestel J, Märk1 B, Schwerer MJ, Arndt A, Kraft K, Pröpper C and Möller P: Expression of Abelson interactor 1 (Abi1) correlates with inflammation, KRAS mutation and adenomatous change during colonic carcinogenesis. PLoS One 7: e40671, 2012.

23. Steinestel K, Brüderlein S, Lennerz JK, Steinestel J, Kraft K, Pröpper C, Meineke V and Möller P: Expression and Y435phosphorylation of Abelson interactor 1 (Abi1) promotes tumour cell adhesion, extracellular matrix degradation and invasion by colorectal carcinoma cells. Mol Cancer 13: 145, 2014.

24. Zhang J, Tang L, Chen Y, Duan Z, Xiao L, Li W, Liu X and Shen L: Upregulation of Abelson interactor protein 1 predicts tumor progression and poor outcome in epithelial ovarian cancer. Hum Pathol 46: 1331-1340, 2015.

25. Liu SY, Wu F, Tao YM and Yang LY: Increased expression of Abil in hepatocellular carcinoma and its correlation with poor prognosis of hepatocellular carcinoma. Zhonghua Wai Ke Za Zhi 47: 1732-1735, 2009 (In Chinese).

26. Chen W, Chen L, Cai Z, Liang D, Zhao B, Zeng Y, Liu X and Liu J: Overexpression of annexin A4 indicates poor prognosis and promotes tumor metastasis of hepatocellular carcinoma. Tumour Biol 37: 9343-9355, 2016.

27. Xiao S, Chang RM, Yang MY, Lei X, Liu X, Gao WB, Xiao JL and Yang LY: Actin-like 6A predicts poor prognosis of hepatocellular carcinoma and promotes metastasis and epithelial-mesenchymal transition. Hepatology 63: 1256-1271, 2016.

28. Stradal T, Courtney KD, Rottner K, Hahne P, Small JV and Pendergast AM: The Abl interactor proteins localize to sites of actin polymerization at the tips of lamellipodia and filopodia. Curr Biol 11: 891-895, 2001.

29. Zipfel PA, Bunnell SC, Witherow DS, Gu JJ, Chislock EM, Ring $\mathrm{C}$ and Pendergast AM: Role for the $\mathrm{Abi} /$ wave protein complex in $\mathrm{T}$ cell receptor-mediated proliferation and cytoskeletal remodeling. Curr Biol 16: 35-46, 2006.

30. Ridley AJ: Rho GTPases and cell migration. J Cell Sci 114: 2713-2722, 2001

31. Kumar S, Lu B, Dixit U, Hossain S, Liu Y, Li J, Hornbeck P, Zheng W, Sowalsky AG, Kotula L, et al: Reciprocal regulation of Abl kinase by Crk Y251 and Abil controls invasive phenotypes in glioblastoma. Oncotarget 6: 37792-37807, 2015. 